\title{
A New Cost-Effective Current-Balancing Multi-Channel LED Driver for a Large Screen LCD Backlight Units
}

\author{
Sung-Soo Hong*, Sang-Hyun Lee*, Sang-Ho Cho*, Chung-Wook Roh*, and Sang-Kyoo Han ${ }^{\dagger}$ \\ $\dagger *$ Dept. of Electrical Engineering, Kookmin University, Seoul, Korea
}

\begin{abstract}
A new current-balancing multi-channel LED driver is proposed in this paper. The conventional LED driver system consists of three cascaded power conversion stages and its driver stage has the same number of expensive boost converters as those of the LED channels. On the other hand, the proposed LED driver system consists of two cascaded power stages and its driver stage requires only passive devices instead of expensive boost converters. Nevertheless, all of the currents through multi-channel LEDs can be well balanced. Therefore, it features a smaller system size, improved efficiency, and lower cost. To confirm the validity of the proposed driver, its operation and performance are verified on a prototype for a 46" LCD TV.
\end{abstract}

Key Words: Backlight unit, Capacitor charge balance, Current balance, LED driver, Multi-channel LEDs, PWM dimming

\section{INTRODUCTION}

In recent years, liquid crystal display (LCD) flat panel displays have become one of the fastest growing markets in large screen displays due to various advantages such as low power consumption, long lifespan, low profile and high contrast ratio [1], [2]. Since LCDs are non-emissive display devices, they usually require a backlight unit (BLU) in monitor or TV applications. In the case of conventional LCD applications, multiple fluorescent lamps such as a cold cathode fluorescent lamp (CCFL), an external electrode fluorescent lamp (EEFL) and a flat fluorescent lamp (FFL) have been used as LCD BLU sources. However, an RoHS directive's limited permission of mercury $(\mathrm{Hg})$ use requires that environmentally friendly lamps be employed as backlight sources.

Recently, light-emitting diodes (LEDs) have become one of the most promising candidates for BLUs and other lighting applications. Although the high cost and low light efficiency of LEDs make it difficult for them to be widely adopted as a backlight source, LEDs satisfy the RoHS directive and have many desirable merits such as a long lifespan, wide color gamut and fast response. Moreover, as the light efficiency of LEDs has improved and the cost reduced, LEDs have become a suitable solution for a BLU source.

To implement sufficient luminance for large sized LCD TVs, series-parallel configured multi-channel LEDs are usually adopted. In driving multi-channel LEDs, it is most important to obtain uniform and sufficient luminance despite the

\footnotetext{
Manuscript received Apr. 26, 2010; revised Jun. 9, 2010

† Corresponding Author: djhan @kookmin.ac.kr

Tel: +82-2-910-5467, Fax: +82-2-910-4449, Kookmin Univ.

* Dept. of Electrical Engineering, Kookmin University, Korea
}

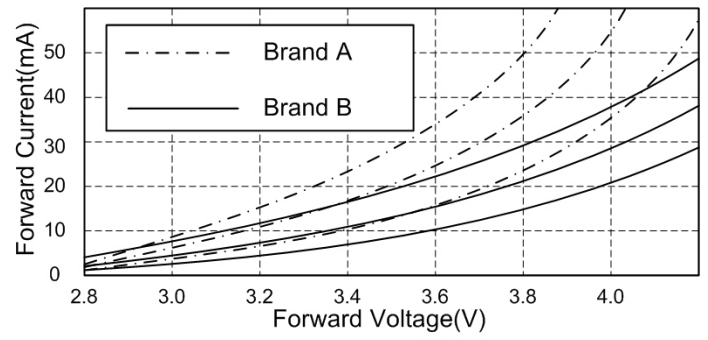

Fig. 1. V-I characteristics of LED.

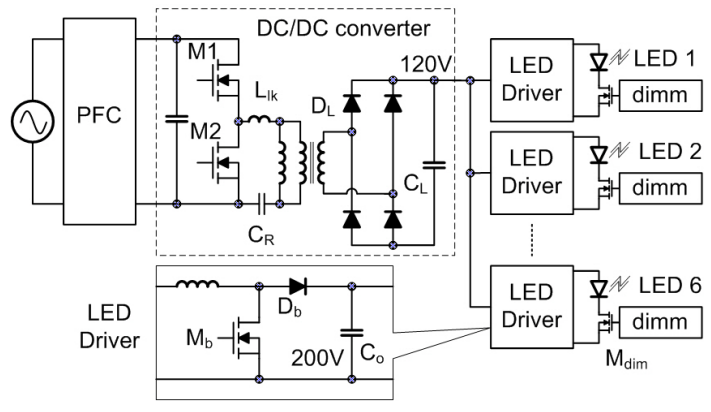

Fig. 2. Schematic of conventional LED driving circuit.

characteristic variations of each LED. Fig. 1 shows the VI characteristics for each LED array produced by different manufacturers. As shown in this figure, each LED has different properties according to the voltage, current and temperature. Thus, although the same voltage is applied to each LED array, there exists LED current variations caused by the temperature of the p-n junction, the time variation and the performance difference of each array [3]-[5]. The variation of each LED 


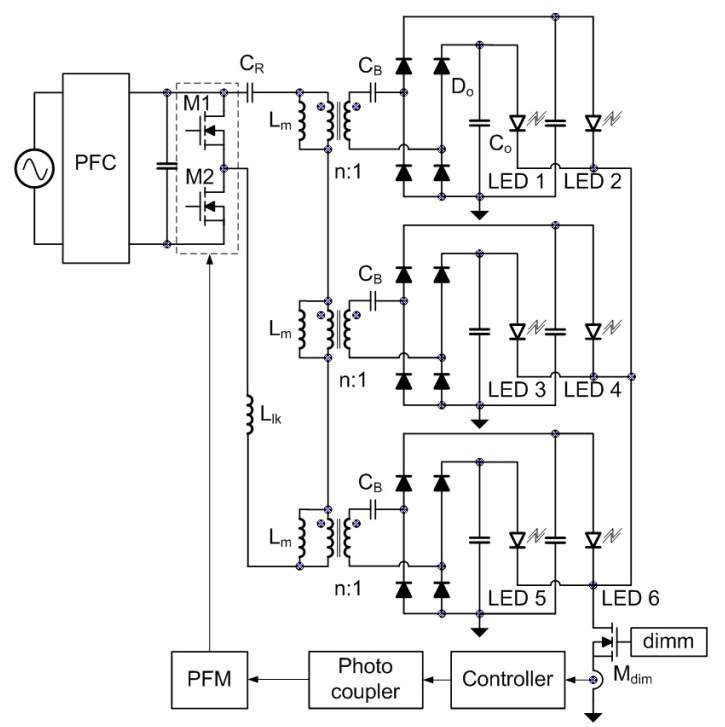

Fig. 3. Schematic of proposed LED driver.

current, proportional to its luminance, could deteriorate its brightness quality, lifespan, and reliability. To overcome the above-mentioned problems, each LED array should be controlled to be balanced by a constant current instead of a constant voltage.

Fig. 2 shows a schematic of a conventional LED driving circuit for a large screen LCD TV. Each LED channel consists of a large number of serially connected LEDs and its operating voltage is about $200 \mathrm{~V}$ in the case of 46" LCD TV. As shown in this figure, the overall system consists of three cascaded power conversion stages, that include a power factor corrector (PFC), an isolated DC/DC converter and a LED driver. The $\mathrm{PFC}$ stage produces a constant voltage from the $\mathrm{AC}$ input voltage source and then the isolated DC/DC converter converts the PFC output voltage into the voltage required to drive the LEDs, where the LLC resonant converter has usually been adopted as an isolated DC/DC converter due to its favorable advantages such as high efficiency and low EMI [6]-[8]. Then, to maintain the currents through the multi-channel LED arrays constant and balanced, one boost converter per LED channel is required to control the current through each LED. Although this driving circuit can tightly control the LED current, it has several disadvantages such as a high cost of production, a low power conversion efficiency, and a bulky system. Moreover, the many active devices and driver ICs used in each boost converter might deteriorate system reliability. This is because the overall system does not consist of three power conversion stages but also the same number of boost converters as those of LED channels.

To overcome above-mentioned drawbacks, a new cost effective current-balancing multi-channel LED driver is proposed as shown in Fig. 3. Since the proposed LED driver requires only passive devices such as transformers and capacitors instead of a boost converter, the cost of production can be considerably reduced and the system reliability can be much improved. At the same time, since the isolated DC/DC converter and LED drivers are integrated into one power stage, the proposed driver system features a high power conversion efficiency and a small size.

\section{A NEW CURRENT BALANCING MULTI-CHANNEL LED DRIVER}

\section{A. Operational principles of the proposed LED driver}

Fig. 3 shows the proposed LED driver, where a LLC resonant inverter is adopted. As can be seen, 3 transformers, 3 capacitors, and 12 diodes are required to drive 6 channel LED arrays. Each transformer's primary side is serially connected with each other and each transformer's secondary side is connected to a DC blocking capacitor $\left(C_{B}\right)$ and a full bridge rectifier. The detailed principles of the current balancing for each LED array are described as follows. For the convenience of the analysis, the following assumptions are made.

1) All parasitic components are discarded except for those specified in Fig. 3.

2) All transformer magnetizing inductors are same as $L_{m}$.

3) The total of the leakage inductors for all of the transformers is $L_{l k}$

4) The voltage across each LED is the same as $V_{L E D}$.

5) From the above assumptions, all the transformer magnetizing currents during each half switching period are equal to $i_{L m_{-} P}$ and $i_{L m_{-} N}$, respectively.

6) The dimming switch is continuously conducting.

Mode 1 : When $M_{2}$ is on and $M_{1}$ is off during a half switching period of the driver, the current through each transformer's primary side is the same as $i_{p r i} N$ as shown in Fig.4 (b). This is because each transformer's primary side is connected in series. Therefore, since all of the transformer magnetizing currents are same as $i_{L m_{-} P}$ from assumption (5) and all of the transformer turn ratios are equal to one another, all of the transformer's secondary side currents are identical as follows:

$\left.\left.\left\langle i_{\text {sec } P 1}\right\rangle=\left\langle i_{\text {sec } P 2}\right\rangle=<i_{\text {sec } P 3}\right\rangle=n<i_{\text {pri } P}-i_{L M P}\right\rangle$

where $\langle\bullet\rangle$ is the average value of ' $\bullet$ '.

Mode 2 : Similarly, when $M_{1}$ is on and $M_{2}$ is off during the other half switching period of the driver, the current through each transformer's primary side is also same as $i_{p r i} P$ as shown in Fig.4 (c). This is because each transformer's primary side is connected in series. Therefore, since all transformer magnetizing currents are the same as $i_{L m_{-} N}$ from assumption (5) and all the transformer turn ratios are equal to one another, all of the transformer's secondary side currents are identical to the same manner of mode 1 as follows:

$\left.\left\langle i_{\text {sec N1 }}\right\rangle=\left\langle i_{\text {sec N2 }}\right\rangle=\left\langle i_{\text {sec N3 }}\right\rangle=n<i_{\text {pri } N}-i_{L M N}\right\rangle$.

At this point, since the blocking capacitor $\left(C_{B}\right)$ is serially connected to the transformer's secondary side, the DC offset current of the transformer's secondary side can be eliminated. Namely, the average current through the transformer's secondary side during each half switching period are equal to one another as follows: 


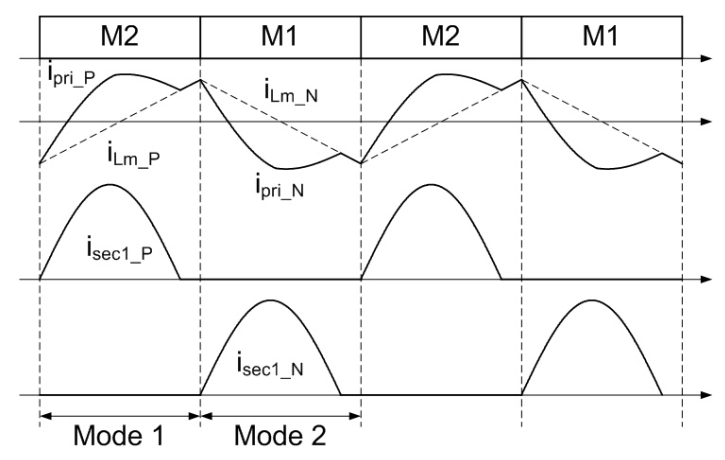

(a) Key waveforms.

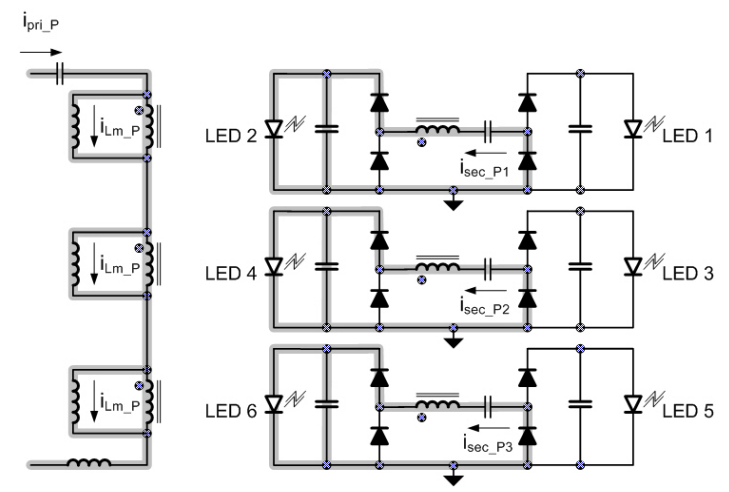

(b) Mode 1 .

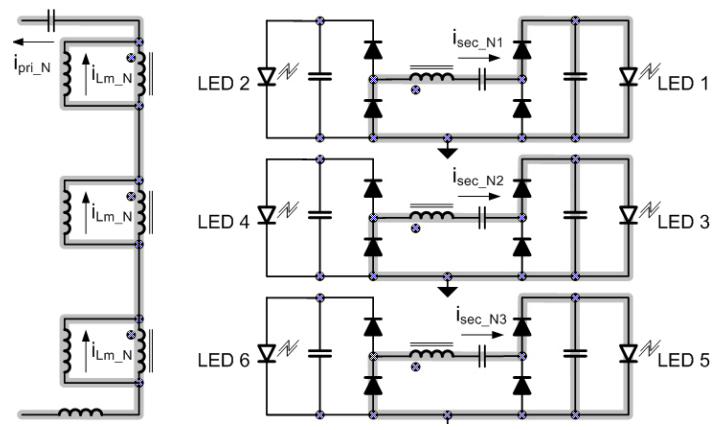

(c) Mode 2.

Fig. 4. Operational mode diagrams.

$$
\begin{aligned}
& <i_{\text {sec } P 1}>=<i_{\text {sec } N 1}>, \\
& <i_{\text {sec } P 2}>=<i_{\text {sec } N 2}>, \\
& <i_{\text {sec } P 3}>=<i_{\text {sec } N 3}>.
\end{aligned}
$$

Finally, from equations (1), (2) and (3), all of the LED currents can be exactly balanced as follows:

$$
\begin{aligned}
& <i_{\text {sec } P 1}>=<i_{\text {sec } N 1}>=<i_{\text {sec } P 2}> \\
& =<i_{\text {sec } N 2}>=<i_{\text {sec } P 3}>=<i_{\text {sec } N 3}>.
\end{aligned}
$$

Accordingly, the proposed driver can keep the currents through all of the LED arrays constant and balanced using only passive devices such as transformers and capacitors.

Moreover, if only the current through one LED array is controlled, all of the currents can be controlled by the above-mentioned principles. Therefore, only one controller is required regardless of the number of LED arrays.
Meanwhile, since the exact same magnetizing inductors and LED voltages can not be obtained, there actually exits a few current of unbalance among each of the LEDs. If the blocking capacitor $\mathrm{CB}$ is large enough to be considered as a constant voltage source $(0 \mathrm{~V})$, the peak current $i_{L m \_p e a k}$ through the transformer magnetizing inductor can be determined by:

$$
i_{L M \_p e a k}=\frac{V_{L E D}}{n L_{m}} \frac{T_{s}}{2}
$$

where $T_{s}$ and $V_{L E D}$ are the switching period and the LED voltage, respectively.

Generally, a larger peak current through the magnetizing inductor produces a larger resonant current and transfers a larger energy to the output side in the LLC resonant converter [6][8]. Therefore, if a magnetizing inductor or LED voltage mismatch exists, differences among the peak magnetizing currents of each transformer arise, which cause a secondary current unbalanced from transformer to transformer and equations (1) and (2) not satisfied anymore. Finally, the LED currents can not be exactly balanced. To mitigate the current unbalance problem caused by the transformer and the LED voltage mismatches, the magnetizing inductor must be designed to be maximized as much as possible, which can minimize the effect of the $V_{L E D}$ variations and the peak current differences

\begin{tabular}{|c|c|c|c|}
\hline \multicolumn{2}{|c|}{ Items } & conventional driver & Proposed driver \\
\hline \multicolumn{2}{|c|}{ transformer } & 1EA (EEM4344) & 3EA (EFD2025) \\
\hline \multirow{3}{*}{$\begin{array}{l}\text { Power } \\
\text { switch }\end{array}$} & M1 \& M2 & 2EA (2SK3561) & 2EA (2SK3561) \\
\hline & $\overline{M_{d i m}}$ & 6EA(FDU3N40) & 1EA(FDU3N40) \\
\hline & $M_{b}$ & 6EA(FDU3N40) & 0EA \\
\hline \multirow[t]{3}{*}{ Diode } & $D_{L L C}$ & 4EA (1N5408) & 0EA \\
\hline & $D_{b}$ & 6EA (UF4007) & 0EA \\
\hline & $\overline{D_{o}}$ & $\overline{\mathrm{OEA}}$ & 12EA (UF4007) \\
\hline \multicolumn{2}{|c|}{ Inductor } & 6EA (500uH) & 0EA \\
\hline \multirow{2}{*}{$\begin{array}{c}\text { Film } \\
\text { capacitor }\end{array}$} & $C_{R}$ & $1 \mathrm{EA}(1 \mathrm{kV})$ & $1 \mathrm{EA}(1 \mathrm{kV})$ \\
\hline & $C_{B}$ & 0EA & $3 \mathrm{EA}(100 \mathrm{~V})$ \\
\hline \multirow{2}{*}{$\begin{array}{l}\text { Electrolytic } \\
\text { capacitor }\end{array}$} & $C_{L}$ & $1 \mathrm{EA}(68 \mathrm{uF} / 200 \mathrm{~V})$ & \\
\hline & $C_{o}$ & $6 \mathrm{EA}(10 \mathrm{uF} / 350 \mathrm{~V})$ & 6EA (10uF/350V) \\
\hline \multirow[t]{2}{*}{ Control IC } & $\overline{\text { LLC }}$ & $\begin{array}{c}\text { 1EA (MC33067) } \\
\text { fsw }=60 \mathrm{kHz}\end{array}$ & $\begin{array}{c}\text { 1EA (MC33067) } \\
\text { fsw }=60 \mathrm{kHz}\end{array}$ \\
\hline & Boost & $\begin{array}{c}\text { 6EA (HV9911) } \\
\text { fsw }=100 \mathrm{kHz}\end{array}$ & 0EA (HV9911) \\
\hline
\end{tabular}
among each magnetizing inductor. Moreover, a transformer with a similar magnetizing inductance must be employed.

TABLE I

COMPARISONS BETWEEN CONVENTIONAL AND PROPOSED LED DRIVERS IN THE ASPECT OF THE NUMBER OF DEVICES

Table I shows a comparison between the conventional and the proposed LED drivers in terms of the number of devices. As shown in this table, since the proposed LED driver can remove many of the expensive devices such as power switches, inductors and control ICs of the conventional inverter, its production cost can be considerably reduced. On the other hand, the increased number of devices such as transformers, diodes and DC blocking capacitors are not dominant in the production cost when compared with the effect of cost reduction.

\section{B. Dimming of the proposed LED driver}

Dimming is one of the most important functions, along with current-balancing, in a LED BLU for LCD TVs [9][11]. Dimming is used to regulate the luminance and contrast 


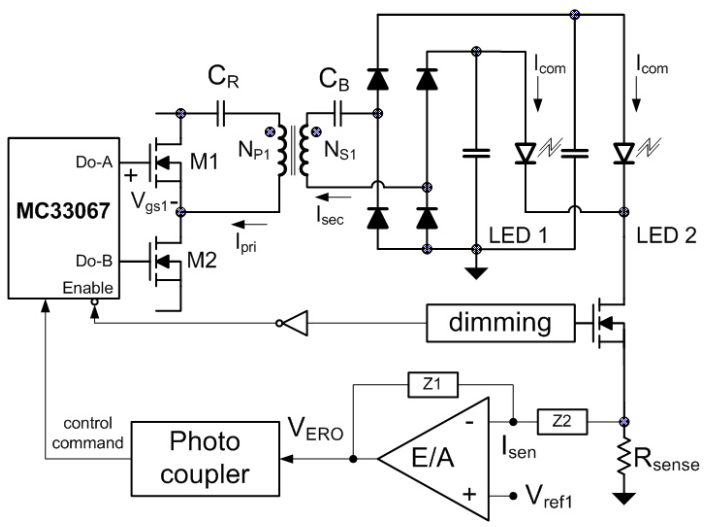

Fig. 5. Block diagram of proposed inverter with PWM dimming circuit.

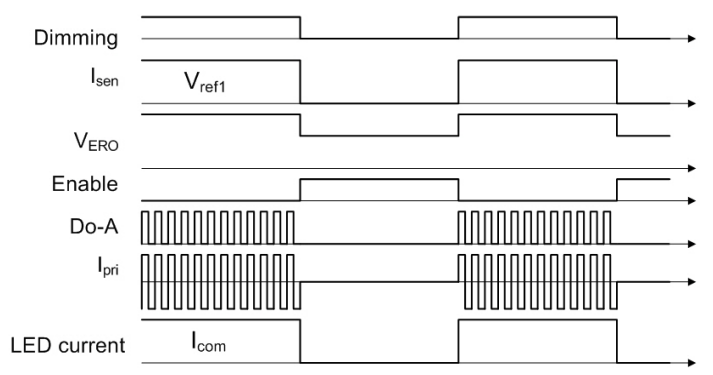

Fig. 6. Key waveforms of PWM dimming.

ratio of a LED BLU according to a user's command or video signal. Its methods can be classified into analog and pulse width modulation (PWM). Firstly, analog dimming is used to regulate the luminance of a BLU by varying the amplitude of the LED current. However, this scheme could generate a color shift in accordance with the amplitude of the LED current and deteriorate the color gamut. On the other hand, PWM dimming is accomplished by regulating the pulse width of the LED current with a current amplitude constant. This dimming scheme allows for a large range of dimming levels. Also, compared with analog dimming, the LEDbrightness intensity of PWM dimming is independent of color, which makes it suitable for many applications [12], [13]. Therefore, the proposed inverter adopts PWM dimming to regulate the brightness as shown in Fig. 5. Fig.6 shows the key waveforms of PWM dimming. As can be seen in this figure, when the dimming signal is high, the switch $M_{\text {dimm }}$ is conducting and the LED current sensed from the sensing resistor $\left(R_{\text {sense }}\right)$ is applied to the inverting input of the error amplifier. Thus, the LED current is controlled to be $I_{\text {com }}$ at the steady state. On the other hand, when the dimming signal is Low, $M_{\text {dimm }}$ is turned off and the inverting input of the error amplifier becomes zero, which is because the LED current is blocked. At the same time, since a high signal is applied to the disable input of the control IC, the inverter is shut down.

\section{EXPERIMENTAL RESULTS}

To confirm the validity of the proposed LED driver, a prototype of a $180 \mathrm{~W}$ rated LED driver for a 46 " LCD TV is implemented with following specifications:

- Inverter input voltage (PFC output voltage) : 385V

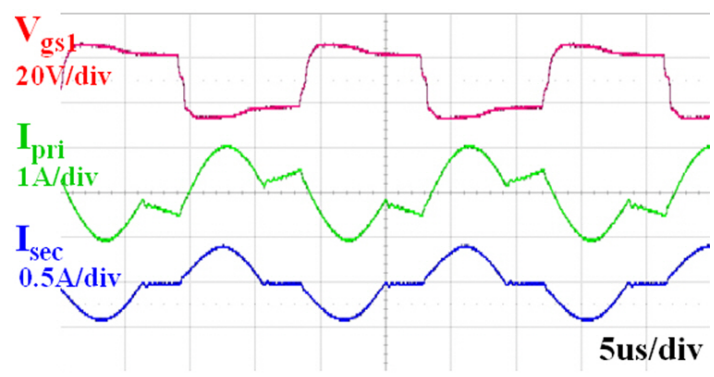

Fig. 7. Key waveforms of proposed LED driver.

- Amplitude of the LED current at the steady state : $100 \mathrm{~mA}$

- The number of LED channels : 6 channels

- Transformer : Core - EFD2025

- Number of turns $N_{p}: N_{s}=29: 74$

- Resonant tank : $L_{m}-700 u H, L_{r}-50 u H, C_{r}-44 n F$

- Output capacitor : $10 u F$

- LED voltage: about $200 \mathrm{~V}$

Fig. 7 shows the key waveforms of the proposed LED driver. As shown in this figure, the current $I_{\text {pri } 1}$ through the transformer's primary side is large enough to ensure ZVS of the main switch $\left(M_{1}, M_{2}\right)$ at the switching instant. Moreover, since the serially connected capacitors $C_{R}$ and $C_{B}$ with the transformer completely block the DC offset current through the transformer magnetizing inductor, the transformer magnetic core can be fully utilized, which can reduce the transformer size and increase the power density.

Fig. 8 shows the experimental waveforms at 20\%, 50\% and $80 \%$ of the dimming ratios. As shown in this figure, the LED current becomes zero at the low dimming signal and it is controlled to be $100 \mathrm{~mA}$ at the high dimming signal. Furthermore, when the dimming signal becomes high, the output voltage and the LED current are slowly increased by soft start, which can reduce the overshoot of the LED current and the acoustic noise.

Table II shows the current balancing performance of each LED array measured using a multi-meter after $30 \mathrm{~min}$. of aging. As shown in this table, the currents through each LED array are almost the same according to the dimming ratios. Fig. 9 shows the linearity of the LED1 current according to the dimming ratio. As shown in this figure, the LED current is linearly increased according to the dimming ratio.

Based on Table I, the power consumption dissipated in the key components averaged over one switching period is shown in fig. 10. Assuming the other components, those not specified in this figure, have similar power consumption and the turn on switching loss of the boost converter is small enough to be discarded due to the DCM (Discontinuous Conduction Mode) operation, the proposed driver shows a lower power loss than the conventional one by about $1.32 \mathrm{~W}$.

Table III shows the comparative results of the measured input power consumption. As shown in this table, the proposed driver system has a smaller power consumption than the conventional system by $1.5 \mathrm{~W}$ under the same conditions. As mentioned above, the conventional LED driver system consists of three cascaded power conversion stages. On the other hand, since the isolated DC/DC converter and the LED drivers are 


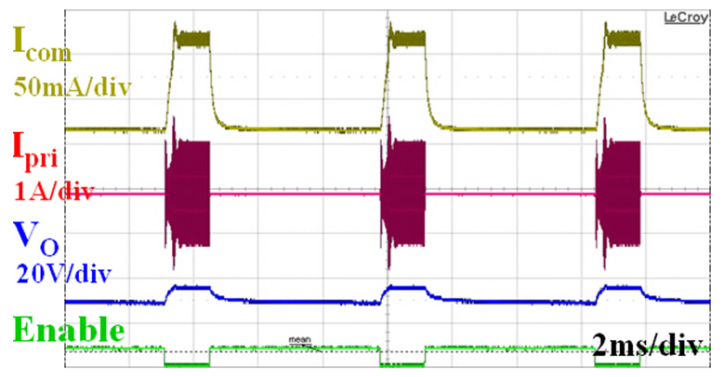

(a) $20 \%$ dimming ratio.

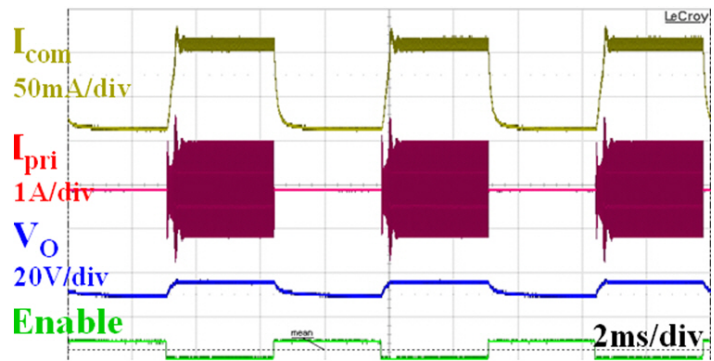

(b) $50 \%$ dimming ratio.

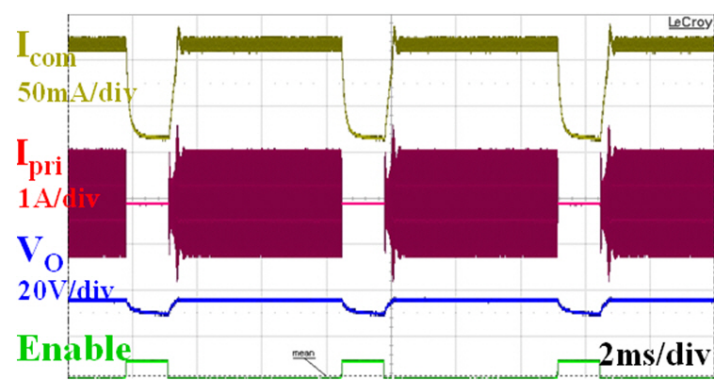

(c) $80 \%$ dimming ratio.

Fig. 8. Experimental waveforms according to dimming ratios.

integrated into one power stage, the proposed LED driver system consists of two cascaded power stages. Therefore, the proposed driver system features better efficiency than the conventional system.

From the above-mentioned experimental results, although the proposed LED driver is composed of several passive devices instead of six expensive boost converters, the current through each LED array can be well controlled to be balanced according to all of the dimming ratios. Therefore, the production cost can be considerably reduced and at the same time, the proposed driver system features higher power conversion efficiency and similar dimming and control performances when compared to the conventional system.

TABLE II

AVERAGE CURRENTS THROUGH LEDS ACCRODING TO DIMMING RATIOS (MA)

\begin{tabular}{|c||c|c|c|c|c|c|}
\hline Dimming & LED1 & LED2 & LED3 & LED4 & LED5 & LED6 \\
\hline \hline $100 \%$ & 100.81 & 101.10 & 100.91 & 100.9 & 101.09 & 101.04 \\
\hline $80 \%$ & 80.86 & 80.89 & 80.47 & 80.45 & 80.77 & 80.86 \\
\hline $60 \%$ & 60.44 & 60.51 & 60.3 & 60.28 & 60.51 & 60.41 \\
\hline $50 \%$ & 49.94 & 50.06 & 49.96 & 50.03 & 50.06 & 50.03 \\
\hline $40 \%$ & 39.92 & 40.05 & 40.02 & 40.03 & 40.04 & 39.98 \\
\hline $20 \%$ & 19.98 & 20.01 & 20.01 & 20.02 & 20.03 & 19.97 \\
\hline
\end{tabular}


TABLE III

COMPARISONS OF MEASURED INPUT POWER CONSUMPTION

\begin{tabular}{|c||c|c|}
\hline Item & Conventional driver system & Proposed driver system \\
\hline \hline Input Power & $185.5 \mathrm{~W}$ & $184.0 \mathrm{~W}$ \\
\hline
\end{tabular}

(Test conditions: $V_{i n}=230 V_{r m s}(A C 60 H z), I_{L E D}=100 m A$, dimming ratio $=100 \%$, measured after $30 \mathrm{~min}$. aging)

such as digital displays, lighting and so on.

\section{ACKNOWLEDGMENT}

This research was supported by the research program 2010 of Kookmin University in Korea and the MKE (Ministry of Knowledge Economy), Korea, under the ITRC (Information Technology Research Center) support program supervised by the NIPA (National IT Industry Promotion Agency) (NIPA2010-C1090-1021-0005).

\section{REFERENCES}

[1] L. Y. Pan, S. C. Chang, M. Y. Liao, and Y. T Lin, "The future development of global LCD TV industry," in Proceeding of PICMET, pp. 1818 1821, Aug. 2007.

[2] F. Xiaoyun, L. Xiaojian, and W. Yan, "Research and analysis of the design development and perspective technology for LED lighting products," in Proceeding of CAID\&CD, pp. 1330-1334, Nov. 2009.

[3] M. Doshi and R. Zane, "Digital architecture for driving large LED arrays with dynamic bus voltage regulation and phase shifted PWM," in Proceeding of APEC, pp.287-293, Mar. 2007.

[4] Heinz Van der Broect, Georg Sauerlander, and Matthias Went, "Power driver topologies and control schemes for LEDs," in Proceeding of APEC, pp.1319-1325, Mar. 2007.

[5] Baddela, S. M. and Zinger, D. S, "Parallel connected LEDs operated at high to improve current sharing," in Proceeding of IAS, Vol. 3, pp.16771681, Oct. 2004.

[6] Roberts W. Erickson and Dragan Maksinmovic, "Fundamentals of power electronics; 2nd edition," Kluwer Academic, pp.705-721, 2001.

[7] Bo Yang Lee, F. C, Zhang, A. J, and Guisong Huang, "LLC resonant converter for front end DC/DC conversion," in Proceeding of APEC, Vol. 2, pp.1108-1112, Mar. 2002.

[8] Jee-Hoon Jung, Jong-Moon Choi, and Joong-Gi Kwon, "Design methodology for transformers including integrated and center-tapped structures for LLC resonant converters," IEEE Transaction on Journal of Power Electronics, Vol. 9, No. 2, pp. 215-223, 2009.

[9] Lee S.T.S., Hui S.Y.R., and Chung H.S.-H, "Practical evaluation of dimming control methods for electronic ballasts," IEEE Transaction on Power Electronics, Vol. 21, Issue 6, pp.1769-1775, Nov. 2006.

[10] S. W. Lee, D. Y. Ko, D. Y. Huh, and Y. I. Yoo, "Simplified control technique for LCD backlight inverter systems using the mixed dimming method," in Proceeding of APEC, pp.447-453, Mar. 2001.

[11] D. Gacio, J.M. Alonso, L. Campa, M. Crespo, and M. Rico-Secades, "High frequency PWM dimming technique for high power factor converters in LED lighting," in Proceeding of APEC, pp. 743-749, Feb. 2010.

[12] Prathyusha Narra and Zinger. D. S, "An effective LED dimming approach," in proceeding of IAS, Vol.3, pp.1671-1676, Oct. 2004.

[13] J. Garcia, A. J. calleja, E.L. Corominas, D. Gacio and J. Ribas, "Electronic driver without electrolynic capacitor for dimming High Brightness LEDs," in Proceeding of IECON, pp. 3518-3523, Nov. 2009.

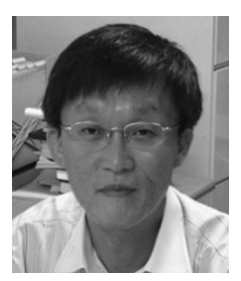

Sung-Soo Hong received his B.S. in Electrical Engineering from Seoul National University, Seoul, Korea, in 1980, and his M.S. and Ph.D. in Electrical Engineering from the Korea Advanced Institute of Science and Technology (KAIST), Daejeon, Korea, in 1986 and 1992, respectively. From 1994 to 1998, he was an Electronics Engineer with Hyundai Electronics Company. In 1993, he was with the Virginia Polytechnic Institute and State University, Blacksburg, as a Research Scientist. Since 1999, he has been an Associate Professor in the Electronics Engineering Department, Kookmin University, Seoul, Korea, His current research interests are in the areas of modeling and control techniques for power converters as well as EMI analysis and reduction techniques for power electronics circuits.

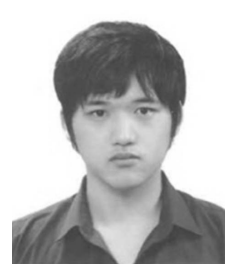

Sang-Hyun Lee received his B.S. in Electronic Engineering from Kookmin University, Seoul, Korea, in 2008, where he is currently working toward his M.S His current research interests are in the areas of analysis, modeling, design, and control of power converters.

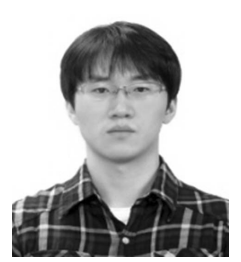

Sang-Ho Cho received his B.S. and M.S. in Electronic Engineering from Kookmin University, Seoul, Korea, in 2006, and 2008, respectively, where he is currently working toward his Ph.D. His current research interests are in the areas of analysis, modeling, design, and control of power converters.

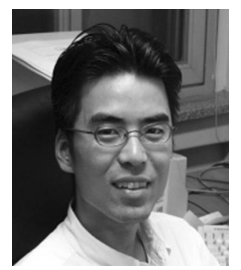

Chung-Wook Roh was born in Busan, Korea, in 1971 He received his B.S., M.S., and Ph.D. in Electrical Engineering from the Korea Advanced Institute of Science and Technology (KAIST), Daejeon, Korea, in 1993, 1995, and 2000, respectively. In 2000, he joined the Digital Media Network Division, Samsung Electronics Company, Suwon, Korea, where he was a Project Leader of the Plasma Display Driver Development Team. Since 2004, he has been an Associate Professor in the Electronics Engineering Department, Kookmin University, Seoul, Korea. His current research interests include driver circuits for plasma display panels, low-power circuits for flat panel displays, modeling, design, and control of power conversion circuits, soft-switching power converters, resonant inverters, and electric drive systems. Dr. Roh is a Member of the Society for Information Display, CA, the Korean Institute of Power Electronics, and the Korean Institute of Electrical Engineers.

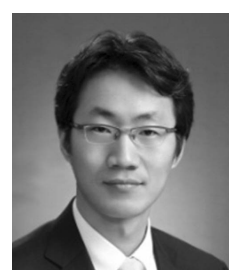

Sang-Kyoo Han was born in Busan, Korea, in 1973. $\mathrm{He}$ received his B.S. in Electrical Engineering from Pusan National University, Busan, Korea, in 1999, and his M.S. and Ph.D. in Electrical Engineering from the Korea Advanced Institute of Science and Technology (KAIST), Daejeon, Korea, in 2001 and 2005, respectively. For six months after that, he worked as a postdoctoral fellow at KAIST. Since September 2005, he has been with Kookmin University, Seoul, Korea, as an Assistant Professor in the Department of Electrical Engineering. He has also been working for the Samsung Power Electronics Center (SPEC) and the Samsung Network Power Center (SNPC) at Kookmin University as a research fellow. His current research interests are in the areas of power converters, renewable energy systems, and display drivers, including analysis, modeling, design, and control. Dr. Han is a member of KIPE and IEEE. 